\title{
Prolyl-4-Hydroxylase $\alpha$ Subunit 2 as a Novel Potential Biomarker for Predicting the Prognosis of Epithelial Ovarian Carcinoma
}

\section{Haibo Zhang (D) \\ Wei Zhao}

Department of Obstetrics and Gynaecology, Hebei Medical University, Fourth Hospital, Shijiazhuang, People's Republic of China
Correspondence: Haibo Zhang Email zhb84021I@163.com
Objective: Epithelial ovarian cancer (EOC) is one of the leading causes of death worldwide. The aim of this study was to explore the prognostic significance of prolyl-4-hydroxylase $\alpha$ subunit 2 (P4HA2) in patients with EOC.

Patients and Methods: A total of 217 clinical samples (EOC tissues, 167 cases; normal ovarian, 50 cases) were collected and pathologically confirmed using hematoxylin and eosin (H\&E) staining. P4HA2 expression in clinical samples was stained by immunohistochemistry (IHC). Relationship between P4HA2 expression and clinicopathological characteristics of EOC patients were analyzed using chi-square test. The differential expression of targets was analyzed in Oncomine database. The prognostic value of P4HA2 was investigated in clinical EOC patients and Kaplan-Meier (KM) Plotter database.

Results: IHC staining showed that P4HA2 was significantly up-regulated in EOC tissues, compared to the normal tissues. Two databases retrieved from Oncomine database further confirmed the up-regulation P4HA2 in EOC. Chi-square test demonstrated that P4HA2 expression was associated with clinical stage $(p=0.036)$, tumor grade $(p<0.001)$, and residual disease $(p=0.022)$. Both in clinical samples and KM Plotter database, high P4HA2 expression was significantly associated with worse progression-free survival (PFS) and overall survival (OS). Cox's proportional hazards regression analysis suggested that high P4HA2 expression were independent risk factors for the survival of EOC patients. Besides, we confirmed the positive correlation between P4HA2 and COL1A1 expression. Moreover, COL1A1 was found to be up-regulated in EOC and also associated with short PFS and OS. Conclusion: The present study preliminarily proved that P4HA2 expression was associated with clinical outcome in EOC patients. P4HA2 might be a prognostic factor for EOC progression, and has the potential to be a valuable therapeutic target for EOC.

Keywords: P4HA2, prognosis, epithelial ovarian cancer, extracellular matrix, collagen

\section{Introduction}

Epithelial ovarian cancer (EOC) is one of the most common gynecological malignancies, and the leading cause of death worldwide. ${ }^{1,2}$ Because there are no symptoms at early stage, the vast majority of EOC patients are already in an advanced stage and have extensive metastasis at the time of diagnosis. ${ }^{3,4}$ Despite significant advancements in the medical technology, diagnosis and treatment for EOC have been achieved over the past two decades, the prognosis of patients has not been significantly improved. ${ }^{5}$ So far, there is no effective treatment for EOC, and the molecular mechanism of its occurrence and development is still not fully understood. Therefore, understanding of 
the pathogenic mechanisms and prognostic factor for EOC progression, and exploring the valuable therapeutic targets for EOC would serve to expand the clinical benefit to a broader patient population.

Proline 4-hydroxylase a subunit 2 (P4HA2) gene is located on chromosome 5q31.1. ${ }^{6}$ As a key enzyme in collagen biosynthesis, P4HA2 plays an important role in maintaining the stability of collagen fiber trimer. Relevant literatures have reported that the high expression level of P4HA2 was associated with the clinical prognosis of many malignant tumors. ${ }^{7-11}$ However, its prognostic value in EOC has not been fully elucidated.

In this study, we found that P4HA2 was markedly upregulated in EOC and the high expression of P4HA2 predicted poor clinical outcomes. Consistent results were observed in the EOC cohorts downloaded from Oncomine and Kaplan-Meier (KM) Plotter database. Moreover, we preliminarily showed that P4HA2 may have the correlation with collagen biosynthesis. Collectively, our results demonstrated that P4HA2 might be a prognostic factor for EOC progression, and has the potential to be a valuable therapeutic target for EOC.

\section{Patients and Methods}

\section{Patients and Tissue Specimens}

A total of 217 paraffin-embedded pathological specimens were collected from the Fourth Affiliated Hospital of Hebei Medical University between September 2000 and June 2014. Pathological diagnosis of EOC were confirmed independently by two pathologists from the Department of Pathology in the Fourth Affiliated Hospital of Hebei Medical University. Among them, there were 104 cases of high-grade serous ovarian cancer (HGSOC), 63 other pathological types (clear cell, mucinous, and endometrioid), and 50 normal ovarian tissues from the patients who had no fertility requirement, and underwent hysterectomy plus bilateral adnexal resection due to cervical lesions. All patients with EOC were staged according to the International Federation of Gynecology and Obstetrics (FIGO) criteria. All participants received the whole treatment and were followed up in our hospital. This study was approved by the ethics committee of the Fourth Hospital of Hebei Medical University, and all participants signed informed consent to participate in this study.

\section{Hematoxylin and Eosin (H\&E) Staining}

Paraffin-embedded sections were stained with H\&E according to the standard protocol. Briefly, paraffin section was dewaxed in xylene, rehydrated in $70-100 \%$ graded alcohol solutions, and washed with twice with $\mathrm{ddH}_{2}$ O. Then, section was stained with hematoxylin and eosin in sequence and were observed under light microscope at appropriate magnification.

\section{Immunohistochemical (IHC) Staining}

IHC staining of paraffin-embedded tissue sections was processed according to standard protocol. Primary antibodies against P4HA2 (Cat No. 66,604-1-Ig, 1:400 dilution) was purchased from Proteintech (Rosemont, IL, USA).

The results of IHC staining were independently determined by two senior pathologists who were blind to the patients' information. If there was any objection, the third pathologist made the judgment. Each pathological section was observed under Olympus BX-51 microscope (Olympus, Waltham, MA, USA). After positively stained cells count and background evaluation, semi-quantitative P4HA2 expression analysis by IHC was evaluated using a staining score, as Gong et al previously described. ${ }^{12}$ The established staining score (range, 0-7) was the sum of staining intensity and proportion of positively stained cells. A final score of $0-4$ points indicated no or low expression, and 5-7 points indicated high expression.

\section{Oncomine Analysis}

The individual gene expression level of P4HA2 and COL1A1 was analyzed in Oncomine database (https:// www.oncomine.org). The P4HA2 related data sets were collected according to the following Oncomine screening conditions: (1) Gene name, P4HA2 or COL1A1; (2) Tissue comparison, ovarian cancer vs normal ovarian tissue; (3) data type, mRNA; (4) Significance: P<1E-4; (5) Differential expression level: more than 2-fold change; (6) Threshold: top $10 \%$ gene rank. Following screening, two datasets (Hendrix database ${ }^{13}$ and Adib database ${ }^{14}$ ) were included for P4HA2 expression analysis and one TCGA dataset, the Cancer Genome Atlas-Ovarian Serous Cystadenocarcinoma Gene Expression Data set, was included for COL1A1 expression analysis.

\section{KM Plotter Database Analysis}

KM plotter integrative data analysis tool (http://www. kmplot.com) was used to analyze the prognostic 
significance of mRNA in patients with ovarian cancer. To assess prognostic significance of genes, the patient samples were divided into two cohorts (high vs low expression) according to auto select best cutoff. Genes (P4HA2 and COL1A1) were retrieved in the platform database respectively to obtain the $\mathrm{KM}$ survival plots, without restriction on the pathological type, clinical stage, and tumor grade. The desired Affymetrix ID (P4HA2, 202733_at; COL1A1, 202311_s_at) ${ }^{15}$ in ovarian cancer is valid in KM plotter database, www.kmplot.com.

\section{Statistical Analysis}

Statistical analysis was performed with the SPSS 21.0 software package (SPSS, Inc., Chicago, IL, USA) and GraphPad Prism (version 8.0; GraphPad Software Inc., CA, USA). $\mathrm{P}<0.05$ (two-sided) was considered significant. The relationship between the expression level of P4HA2 and the clinicopathological characteristics was analyzed by chi-square test. Survival curves were plotted using the Kaplan-Meier method and compared using the Log rank test. Influence factors for survival were evaluated using univariate and multivariate Cox regression analysis. The correlation between P4HA2 and COL1A1 expression was analyzed using Spearman correlation.

\section{Results}

\section{P4HA2 Expression in Patients with EOC}

In this study, the EOC tissue $(n=167)$ and normal ovarian tissues $(n=50)$ were pathologically confirmed using H\&E staining (Figure 1A). To investigate the role of P4HA2 in EOC, IHC staining was performed, which confirmed the up-regulation P4HA2 in EOC tissues, compared to the normal tissues (Figure $1 \mathrm{~B}$ and $\mathrm{C}$ ). To verify the clinical finding, three dataset was retrieved from Oncomine database (Figure 2). The results showed that the levels of P4HA2 in EOC were higher than that in normal tissues, in all three datasets including Adib dataset $(p=0.002$, Figure 2A), and Hendrix dataset ( $p<0.001$, Figure 2B). Therefore, these above results confirmed the upregulation of $\mathrm{P} 4 \mathrm{HA} 2$ in patients with $\mathrm{EOC}$, indicating the potential role of $\mathrm{P} 4 \mathrm{HA} 2$ in EOC progression.

\section{Relationship Between P4HA2 and Clinicopathologic Characteristics of Patients with EOC}

To investigate the association of P4HA2 with EOC progression, we analyzed the relationship between P4HA2 expression and clinicopathological characteristics of the patients with EOC. As shown in Table 1, the P4HA2 expression had no significant association with the age $(p=0.956)$ and clinical histology $(p=0.203)$ of EOC patients. Notably, the high expression of P4HA2 was significantly associated with clinical stage $(p=0.036)$, grade $(p<0.001)$, and residual disease $(p=0.022)$.

\section{P4HA2 Expression is Associated with Poor Prognosis in Patients with EOC}

Subsequently, to explore the influence of P4HA2 expression on patients' survival, KM survival analysis was performed in clinical samples and $\mathrm{KM}$ plotter database, respectively. As shown in Figure 3A and B, the high
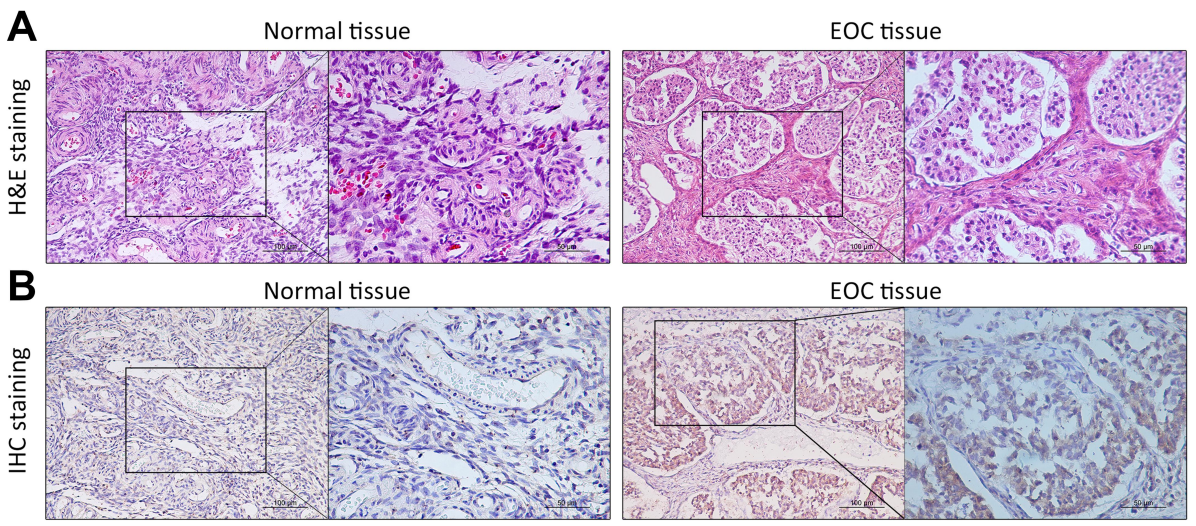

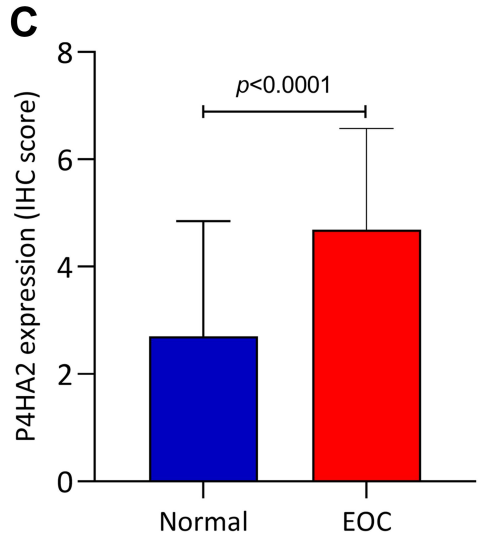

Figure I The up-regulation of P4HA2 in clinical samples of patients with EOC. (A) The normal ovarian tissues and EOC tissue were pathologically confirmed using H\&E staining. Representative images were shown at 200x magnification (scale bar, $100 \mu \mathrm{m}$ ) and 400x magnification (scale bar, $50 \mu \mathrm{m}$ ). (B) The expression of P4HA2 in normal ovarian tissues and EOC tissue were detected using IHC staining. Representative images were shown at $200 \times$ magnification (scale bar, $100 \mu \mathrm{m}$ ) and $400 \times$ magnification (scale bar, $50 \mu \mathrm{m}$ ). (C) Semi-quantitative P4HA2 expression in normal ovarian tissues and EOC tissue by staining score. Abbreviation: EOC, epithelial ovarian cancer. 


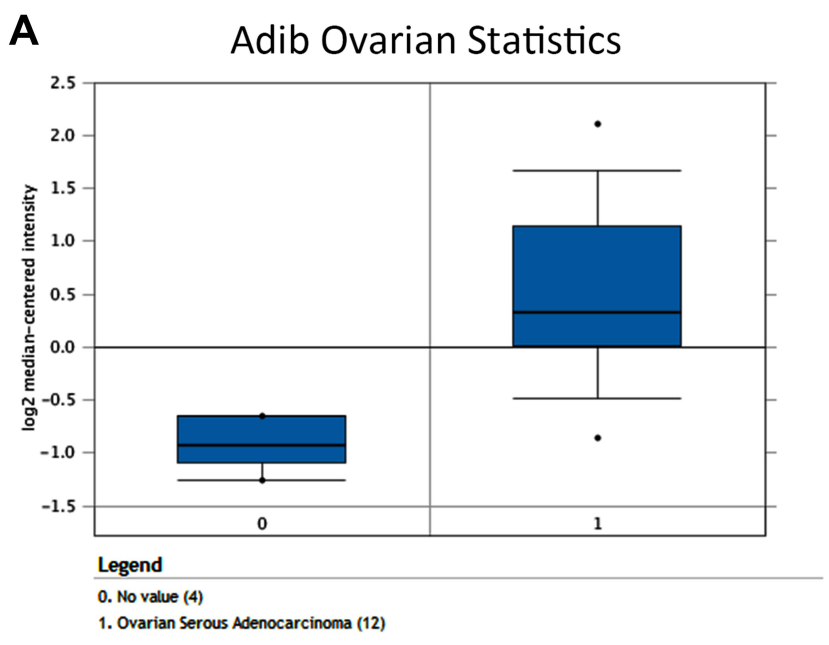

Figure 2 The up-regulation of $\mathrm{P} 4 \mathrm{HA} 2$ in EOC patients from Oncomine database. P4HA2 mRNA in Hendrix ovarian dataset.

Abbreviation: EOC, epithelial ovarian cancer.

P4HA2 expression is significantly associated with worse PFS ( $p=0.0093$, Figure $3 \mathrm{~A})$ and OS $(p=0.0374$, Figure 3B) in clinical samples. Through the KM plotter integrative data analysis, we further verified that high P4HA2 levels predicted PFS $(p<0.001$, Figure $3 \mathrm{C})$ and OS $(p<0.001$, Figure 3D) in EOC, which consistent with the findings from clinical samples. Furthermore, to verify the influence of P4HA2 expression on patients' survival, Cox's
B Hendrix Ovarian Statistics

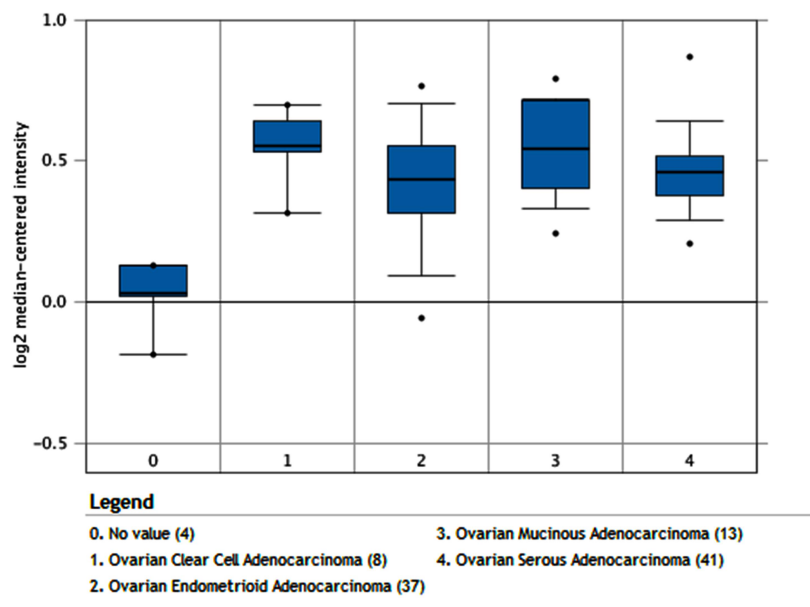

proportional hazards regression model was performed by adjusting clinical parameters (Table 2). Both the univariate and multivariate analysis showed that high clinical stage (III-IV), large residual disease $(>1 \mathrm{~cm})$, and high P4HA2 expression were independent risk factors for the survival of patients with EOC. Moreover, as HGSOC is the most common type of ovarian cancer, we compared the expression of P4HA2 in HGSOC with other pathological types.

Table I Association Between P4HA2 Expression and Clinicopathologic Characteristics of EOC Patients

\begin{tabular}{|c|c|c|c|c|}
\hline \multirow[t]{2}{*}{ Characteristics } & \multirow[t]{2}{*}{ Cases } & \multicolumn{3}{|c|}{ P4HA2 Expression } \\
\hline & & Low Expression & High Expression & $P$-value \\
\hline \multicolumn{5}{|l|}{ Age (years) } \\
\hline$<50$ & 78 & 47 & 31 & 0.956 \\
\hline$\geq 50$ & 89 & 54 & 35 & \\
\hline \multicolumn{5}{|l|}{ Clinical stage } \\
\hline I-II & 59 & 42 & 17 & 0.036 \\
\hline III-IV & 108 & 59 & 49 & \\
\hline \multicolumn{5}{|l|}{ Grade } \\
\hline GI & 41 & 34 & 7 & $<0.001$ \\
\hline G2-G3 & 126 & 67 & 59 & \\
\hline \multicolumn{5}{|l|}{ Clinical histology } \\
\hline Serous & 104 & 59 & 45 & 0.203 \\
\hline Others & 63 & 42 & 21 & \\
\hline \multicolumn{5}{|l|}{ Residual disease $(\mathrm{cm})$} \\
\hline$\leq 1$ & 66 & 47 & 19 & 0.022 \\
\hline$>1$ & 101 & 54 & 47 & \\
\hline
\end{tabular}



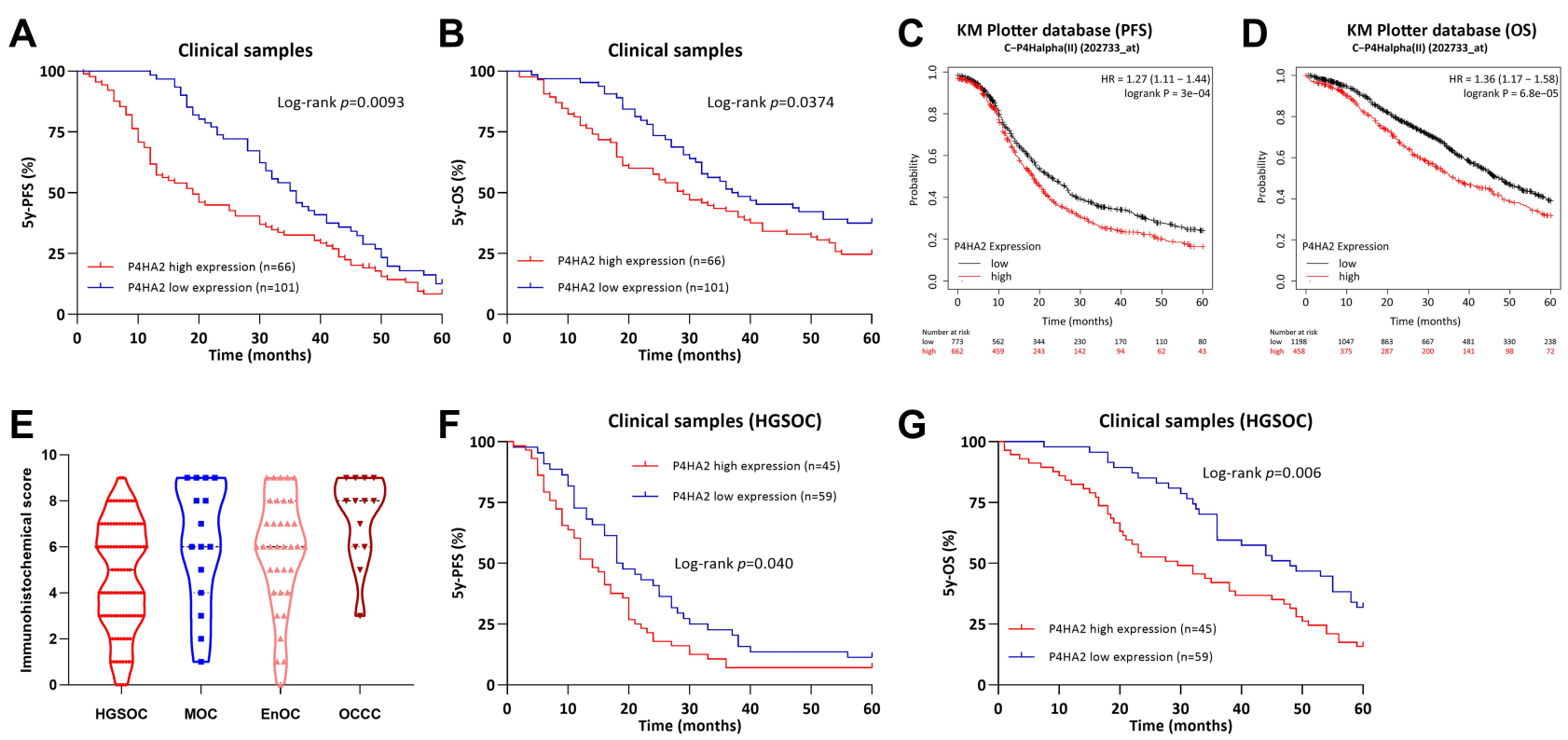

Figure 3 P4HA2 expression is associated with poor prognosis in patients with EOC. (A and B) Kaplan-Meier curves of progression-free survival (A) and overall survival (B) in EOC patients categorized by P4HA2 expression; (C and D) the Kaplan-Meier plotter platform (http://www.kmplot.com) resulted that patients with the high P4HA2 expression was significantly associated with short progression-free survival (C) and poor overall survival (D) in EOC; (E) The P4HA2 expression in different pathological types. (F and $\mathbf{G})$ Kaplan-Meier curves of progression-free survival (F) and overall survival (G) in HGSOC patients categorized by P4HA2 expression.

Abbreviations: EOC, epithelial ovarian cancer; HGSOC, high-grade serous ovarian cancer; MOC, mucinous ovarian cancer; EnOC, endometrioid ovarian cancer; OCCC, ovarian clear cell carcinoma.

As shown in Figure 3E, there was no significant difference in the expression of P4HA2 among different pathological types of EOC. In the patients with HGSOC, the analysis of clinical samples showed that the high P4HA2 expression is also significantly associated with worse PFS ( $\mathrm{p}=0.040$, Figure $3 F)$ and $O S(p=0.006$, Figure $3 G)$. Overall, these results suggested that $\mathrm{P} 4 \mathrm{HA} 2$ expression is associated with poor prognosis in patients with EOC.

COL1A1 is often found to be associated with metastatic HGSOC, which is the most common type of ovarian cancer, thus we investigated the relationship of P4HA2 with COL1A1. As expected, COL1A1 was also upregulated in EOC from Oncomine database (Figure 4A). More importantly, P4HA2 expression was found to be positively correlated with COL1A 1 expression $(\mathrm{R}=0.43$, $p<0.001$, Figure 4B). Similarly, KM plotter integrative data analysis indicated that high COL1A1 expression is significantly associated with worse PFS ( $p=0.0093$, Figure $4 \mathrm{C})$ and $\mathrm{OS}(p=0.0374$, Figure $4 \mathrm{D})$. These results provided additional evidence for the role of P4HA2 in EOC.

\section{Discussion}

EOC is a significant cause of cancer related morbidity and mortality in women. ${ }^{16}$ Due to the special anatomic location of the ovary, the disease is usually lack of clinical symptoms in the early stage, leading to the poor outcome for patients in advanced stage. ${ }^{17}$ There are many mechanisms are responsible for the development and progression of EOC, including cell proliferation, ${ }^{18}$ apoptosis inhibition, ${ }^{19}$ tumor stroma formation, ${ }^{20}$ and peripheral

Table 2 Cox Regression Analysis for Overall Survival in Patients with EOC

\begin{tabular}{|c|c|c|c|c|c|c|}
\hline \multirow[t]{2}{*}{ Characteristics } & \multicolumn{3}{|c|}{ Univariate Analysis } & \multicolumn{3}{|c|}{ Multivariate Analysis } \\
\hline & HR & $95 \% \mathrm{Cl}$ & $P$-value & HR & $95 \% \mathrm{Cl}$ & $P$-value \\
\hline Age ( $<50$ vs $\geq 50$ years) & 1.387 & $0.912-2.110$ & 0.126 & 1.485 & $0.948-2.324$ & 0.084 \\
\hline Clinical stage (III-IV vs I-II) & 1.514 & $1.236-1.855$ & $<0.001$ & 1.29 & $1.017-1.636$ & 0.036 \\
\hline Grade (G2-G3 vs GI) & 0.925 & $0.720-1.190$ & 0.546 & 0.88 & $0.673-1.151$ & 0.352 \\
\hline Clinical histology (Serous vs others) & 0.976 & $0.767-1.242$ & 0.842 & 0.891 & $0.661-1.201$ & 0.450 \\
\hline Residual disease $(>\mid$ vs $\leq \mid \mathrm{cm}$ ) & 1.858 & $1.392-2.478$ & $<0.001$ & 1.704 & $1.268-2.289$ & $<0.001$ \\
\hline P4HA2 expression (High vs low) & 4.495 & $2.258-8.948$ & $<0.001$ & 3.35 & $1.619-6.934$ & 0.001 \\
\hline
\end{tabular}



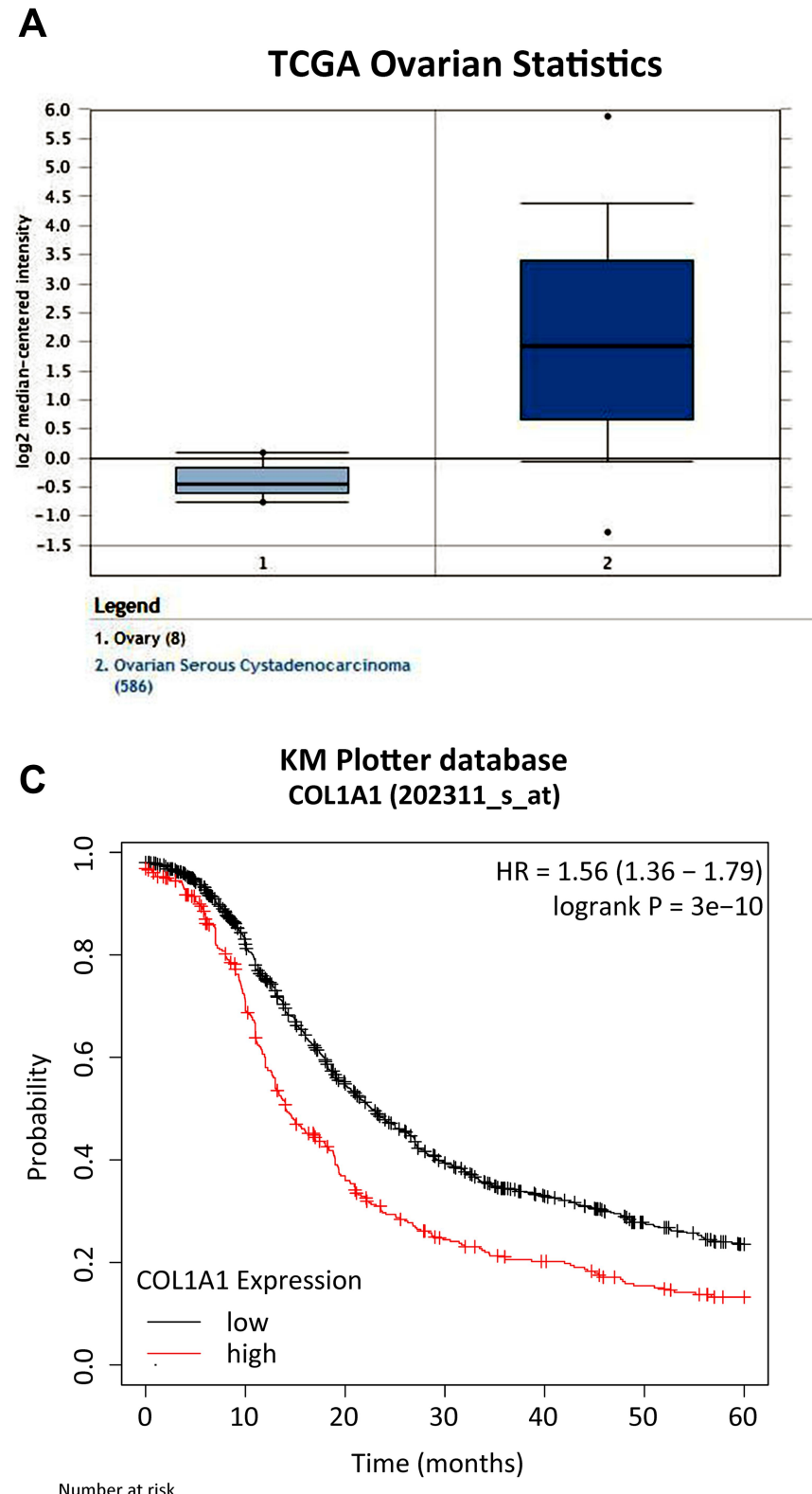

Number at risk

low 1055

high 380
KM Plotter database

Time (months)

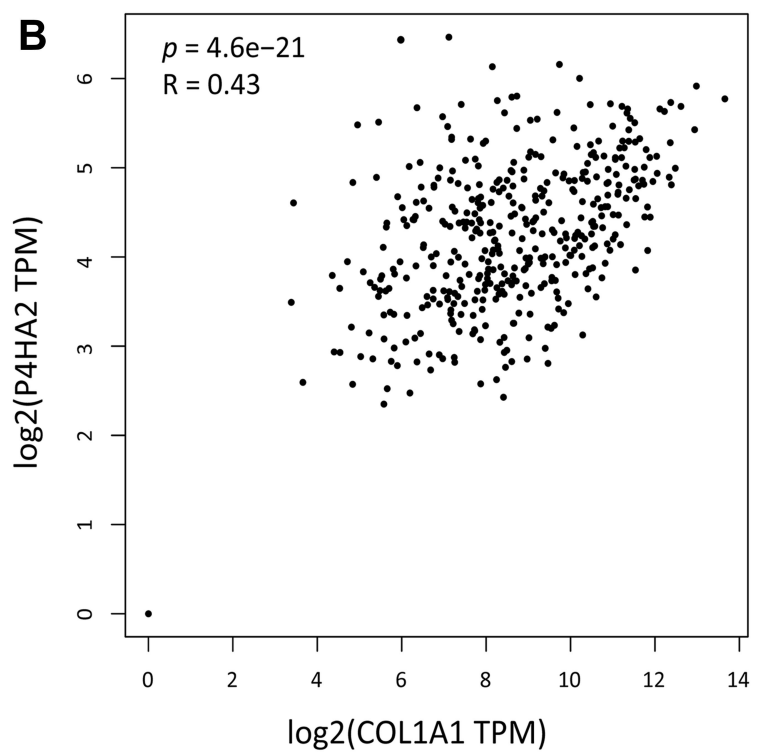

D KM Plotter database

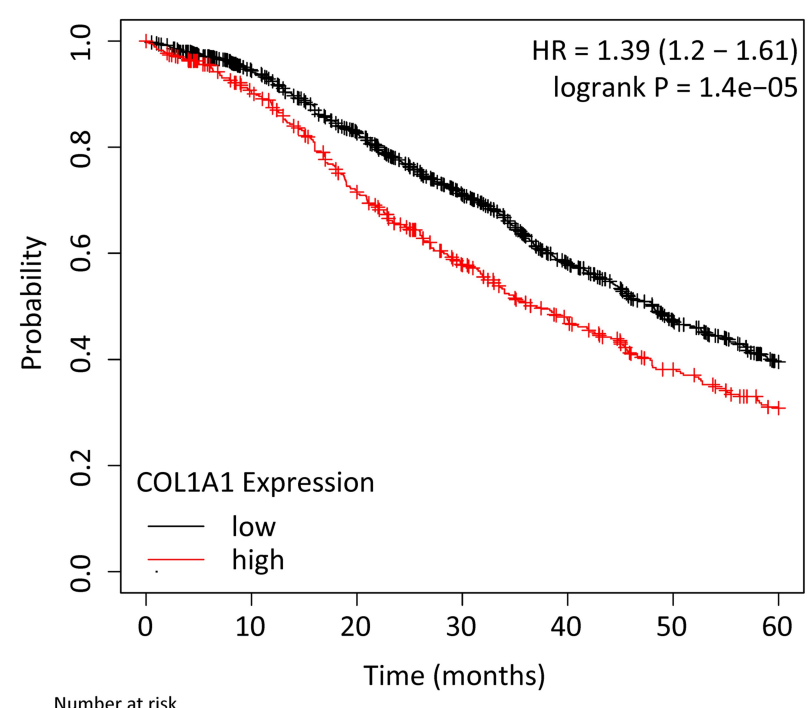

Number at risk

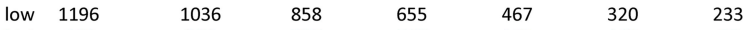

Figure 4 The relationship between P4HA2 with COLIAI. (A) The expression of COLIAI mRNA in TCGA ovarian dataset; (B) the correlation analysis between P4HA2 and COLIAI expression was analyzed using Spearman correlation in the GEPIA database; (C and D) the Kaplan-Meier plotter platform (http://www.kmplot.com) resulted that patients with the high COLIAI expression was significantly associated with short progression-free survival (C) and poor overall survival (D) in EOC.

Abbreviation: EOC, epithelial ovarian cancer.

blood vessels. ${ }^{21}$ Although these aspects are regulated by various factors, there are still lack of effective biomarkers and targets to guide clinical treatment. Therefore, it is very important to identify novel prognostic biomarkers and therapeutic targets in EOC.

In this study, the association between the P4HA2 expression and the clinical outcomes of EOC patients was investigated. The results showed that the expression levels of P4HA2 were statistically up-regulated in
EOC tissue compared to normal ovarian tissues. Meanwhile, univariate and multivariate analysis demonstrated that patients with high expression levels of P4HA2 had a worse prognosis, indicating that P4HA2 might be act as an independent prognostic factor in EOC progression. This finding was consistent with the previous studies, including melanoma ${ }^{7}$ cervical cancer, ${ }^{8}$ liver cancer, ${ }^{9}$ lung cancer, ${ }^{10}$ and breast cancer. ${ }^{11}$ 
Many previous studies have shown that extracellular matrix (ECM) is important for the occurrence and development of malignancies. ${ }^{22,23}$ Moreover, collagen is an important part of ECM and forms the scaffold of tumor micro-environment, ${ }^{9}$ thus, is plays a critical role in tumor infiltration and metastasis. ${ }^{24}$ Collagen biosynthesis and deposition are a multi-step process, which regulated by a variety of post-transcriptional modifying enzymes, especially the collagen pro4hydroxylase. ${ }^{25}$ P4HA2 as the member of the collagen pro4-hydroxylase family could increase collagen deposition. ${ }^{11}$ Many previous studies have shown that high expression of P4HA2 is associated with poor prognosis of malignancies. P4HA2 promoted proliferation, and particularly invasiveness in melanoma. ${ }^{7}$ Epithelial-mesenchymal transition (EMT) was involved in cervical cancer progression regulated by P4HA2. ${ }^{8}$ Feng et al have demonstrated that HBxelevated P4HA2 enhanced the collagen deposition in the liver in vivo and in vitro, leading to liver fibrosis and liver cancer progression. ${ }^{9}$ A similar finding was found in lung cancer. ${ }^{10}$ Moreover, increased mRNA levels of P4HA2 correlated with poor clinical outcome in breast cancer patients. ${ }^{11}$ In this study, we confirmed that P4HA2 was highly expressed in EOC and associated with poor prognosis. Moreover, given that HGSOC is the most common type of ovarian cancer, the expression of P4HA2 in HGSOC comparing with other pathological types were analyzed. However, no expression difference was found among different pathological types, indicating that the P4HA2 may be a common biomarker for different pathological types of EOC. Certainly, this speculation still needed to be verified in the further investigation.

COL1A1 was often found to be associated with metastatic HGSOC, which is the most common type of ovarian cancer, thus we investigated the relationship of P4HA2 with COL1A1. As expected, COL1A1 was found to be upregulated in EOC and also associated with poor progression in EOC. More importantly, P4HA2 expression was found to be positively correlated with COL1A1 expression. These results provided additional evidence for the role of P4HA2 in EOC.

In conclusion, the present study preliminarily proved that P4HA2 expression was associated with clinical outcome in EOC patients. P4HA2 might be a prognostic factor for EOC progression, and has the potential to be a valuable therapeutic target for EOC.

\section{Ethical Approval}

This study was approved by the Medical Ethics Committee of the Fourth Hospital of Hebei Medical University. All the procedures were in accordance with the ethical standards of the institutional and/or national research committee and with the 1964 Helsinki Declaration and its later amendments or comparable ethical standards.

\section{Acknowledgments}

We are grateful to the contributors of data to Oncomine and Kaplan-Meier plotter.

\section{Author Contributions}

All authors contributed to data analysis, drafting or revising the article, have agreed on the journal to which the article will be submitted, gave final approval of the version to be published, and agree to be accountable for all aspects of the work.

\section{Funding}

This work was supported by the Scientific Research Fund of Hebei Provincial Health and Family Planning Commission (no.20201091).

\section{Disclosure}

All authors declare that they have no conflict of interest.

\section{References}

1. Jemal A, Siegel R, Xu J, Ward E. Cancer statistics, 2010. CA Cancer J Clin. 2010;60(5):277-300. doi:10.3322/caac.20073

2. Sankaranarayanan R, Ferlay J. Worldwide burden of gynaecological cancer: the size of the problem. Best Pract Res Clin Obstet Gynaecol. 2006;20(2):207-225. doi:10.1016/j.bpobgyn.2005.10.007

3. Armstrong DK. Relapsed ovarian cancer: challenges and management strategies for a chronic disease. Oncologist. 2002;7(Suppl 5):20-28. doi:10.1634/theoncologist.7-suppl_5-20

4. Lan C, Li Y, Liu J. Intraperitoneal access via direct puncture is an alternative way to deliver intraperitoneal chemotherapy in ovarian, fallopian tube and primary peritoneal cancer. Gynecol Oncol. 2009;114(1):42-47. doi:10.1016/j.ygyno.2009.03.034

5. Anuradha S, Webb PM, Blomfield P, et al. Survival of Australian women with invasive epithelial ovarian cancer: a population-based study. Med J Aust. 2014;201(5):283-288. doi:10.5694/mja14.00132

6. Myllyharju J. Prolyl 4-hydroxylases, the key enzymes of collagen biosynthesis. Matrix Biol. 2003;22(1):15-24. doi:10.1016/S0945053X(03)00006-4

7. Atkinson A, Renziehausen A, Wang H, et al. Collagen prolyl hydroxylases are bifunctional growth regulators in melanoma. $J$ Invest Dermatol. 2019;139(5):1118-1126. doi:10.1016/j.jid.2018.10.038

8. Cao Y, Han Q, Li J, Jia Y, Zhang R, Shi H. P4HA2 contributes to cervical cancer progression via inducing epithelial-mesenchymal transition. J Cancer. 2020;11(10):2788-2799. doi:10.7150/jca.38401

9. Feng GX, Li J, Yang Z, et al. Hepatitis B virus X protein promotes the development of liver fibrosis and hepatoma through downregulation of miR-30e targeting P4HA2 mRNA. Oncogene. 2017;36 (50):6895-6905. doi:10.1038/onc.2017.291 
10. Pankova D, Jiang Y, Chatzifrangkeskou M. RASSF1A controls tissue stiffness and cancer stem-like cells in lung adenocarcinoma. EMBO J. 2019;38(13):e100532. doi:10.15252/embj.2018100532

11. Xiong G, Deng L, Zhu J, Rychahou PG, Xu R. Prolyl-4-hydroxylase $\alpha$ subunit 2 promotes breast cancer progression and metastasis by regulating collagen deposition. BMC Cancer. 2014;14:1. doi:10.1186/ 1471-2407-14-1

12. Gong W, Ni M, Chen Z, Zheng Z. Expression and clinical significance of methyl-CpG binding domain protein 2 in high-grade serous ovarian cancer. Oncol Lett. 2020;20(3):2749-2756.

13. Hendrix ND, Wu R, Kuick R, Schwartz DR, Fearon ER, Cho KR. Fibroblast growth factor 9 has oncogenic activity and is a downstream target of Wnt signaling in ovarian endometrioid adenocarcinomas. Cancer Res. 2006;66(3):1354-1362. doi:10.1158/ 0008-5472.CAN-05-3694

14. Adib TR, Henderson S, Perrett C, et al. Predicting biomarkers for ovarian cancer using gene-expression microarrays. $\mathrm{Br} \mathrm{J}$ Cancer. 2004;90(3):686-692. doi:10.1038/sj.bjc.6601603

15. Gyorffy B, Lánczky A, Szállási Z. Implementing an online tool for genome-wide validation of survival-associated biomarkers in ovarian-cancer using microarray data from 1287 patients. Endocr Relat Cancer. 2012;19(2):197-208. doi:10.1530/ERC-11-0329

16. Cress RD, Chen YS, Morris CR, Petersen M, Leiserowitz GS. Characteristics of long-term survivors of epithelial ovarian cancer. Obstet Gynecol. 2015;126(3):491-497. doi:10.1097/ AOG.0000000000000981

17. Henderson BE, Feigelson HS. Hormonal carcinogenesis. Carcinogenesis. 2000;21(3):427-433. doi:10.1093/carcin/21.3.427

18. Liu L, Wang X, Li X, Wu X, Tang M, Wang X. Upregulation of IGF1 by tumor-associated macrophages promotes the proliferation and migration of epithelial ovarian cancer cells. Oncol Rep. 2018;39 (2):818-826. doi:10.3892/or.2017.6148
19. Liu Y, Wang Y, Yao D, Cui D. LncSOX4 serves an oncogenic role in the tumorigenesis of epithelial ovarian cancer by promoting cell proliferation and inhibiting apoptosis. Mol Med Rep. 2018;17 (6):8282-8288. doi:10.3892/mmr.2018.8892

20. Akahane T, Hirasawa A, Tsuda H, et al. The origin of stroma surrounding epithelial ovarian cancer cells. Int J Gynecol Pathol. 2013;32(1):26-30. doi:10.1097/PGP.0b013e3182518533

21. Ghinea N. Vascular endothelial FSH receptor, a target of interest for cancer therapy. Endocrinology. 2018;159(9):3268-3274. doi:10.1210/ en.2018-00466

22. Girigoswami K, Saini D. Extracellular matrix remodeling and development of cancer. Stem Cell Rev Rep. 2020;1-9. doi:10.1007/s12015019-09951-x

23. Walker C, Mojares E, Del Río Hernández A. Role of extracellular matrix in development and cancer progression. Int J Mol Sci. 2018;19 (10). doi:10.3390/ijms19103028

24. Chen D, Chen G, Jiang W, et al. Association of the collagen signature in the tumor microenvironment with lymph node metastasis in early gastric cancer. JAMA Surg. 2019;154(3):e185249. doi:10.1001/ jamasurg.2018.5249

25. Tameda M, Sugimoto K, Shiraki K, et al. Collagen triple helix repeat containing 1 is overexpressed in hepatocellular carcinoma and promotes cell proliferation and motility. Int $J$ Oncol. 2014;45 (2):541-548. doi:10.3892/ijo.2014.2445

\section{Publish your work in this journal}

Cancer Management and Research is an international, peer-reviewed open access journal focusing on cancer research and the optimal use of preventative and integrated treatment interventions to achieve improved outcomes, enhanced survival and quality of life for the cancer patient.
The manuscript management system is completely online and includes a very quick and fair peer-review system, which is all easy to use. Visit http://www.dovepress.com/testimonials.php to read real quotes from published authors. 